transglutaminase (IgA) antibodies. Six patients were DQ2 positive.

After being diagnosed of CD and starting the GFD, SLE patients seem to improve especially the leukopenia, lymphopenia and oral aphtosis, as well as SLEDAI score (showed in attached graphics).

Conclusions SLE patients with CD diagnosis and who started a GFD, showed improvement of leukopenia, lymphopenia, oral aphtosis and even SLEDAI.

In SLE patients with recurrent oral aphtosis and/or gastrointestinal unspecific symptoms, CD should be considered, but since serological screening displays a low sensitivity, HLA testing could be helpful. Gastroscopy should be considered, with biopsy and flow cytometer in uncertain cases. Even though, further studies, especially looking for different clinical profiles and longer observational period are needed.

\section{P62 POLY-AUTOIMMUNITY FREQUENCY IN SLE PATIENTS FROM A TERTIARY HOSPITAL}

Samuel Leal-Rodríguez, Elena Grau-García, Roxana González-Mazarío, Marta De-la-RubiaNavarro, Cristóbal Pávez-Perales, Isabel Martínez-Cordellat, Carmen Nájera-Herranz, Rosa Negueroles-Albuixech, José Eloy Oller-Rodríguez, Francisco Miguel Ortiz-Sanjuán, Elvira Vicens-Bernabeu, Cristina Alcañiz-Escandell, Inés Cánovas-Olmos, Inmaculada Chalmeta-Verdejo, Jorge Juan Fragio-Gil, Luis González-Puig, José Ivorra-Cortés, José Andrés Román-Ivorra. Rheumatology Dept., Hospital Universitario y Politécnico de La Fe, Valencia, Spain

\subsection{6/lupus-2020-eurolupus. 109}

Background/Purpose Poly-autoimmunity (PAI) is the presence of more than one Autoimmune Disease (AID) in one patient. The coexistence of Systemic Lupus Erythematosus (SLE) with other AIDs is a clinical challenge due to is one of the issues not yet elucidated in medical practice.

We aimed to determine PAI frequency in the context of SLE patients reported in a tertiary hospital.

Methods Cross-sectional observational study with systematic revision of electronic clinical records of SLE patients with other AIDs (from 2014 to 2018) was performed. Demographic, clinical and immunological data were collected.

Results Of 261 SLE patients, 48 (18.39\%) had PAI. Mean age was 51.19 (15.35) years $(93.75 \%$ were female). 2 patients from the $48(4.16 \%)$ had PAI with three AIDs. The $75 \%$ of cases developed SLE as the first AID. The mean age at diagnosis of the first AID was 35.52 (15.33) years and mean age at diagnosis of the second AID was 43.75 (16.31) years. A mean difference of 8.31 (9.24) years between the first and second AIDs debut was observed.

The most frequent AIDs registered that go along with SLE are Antiphospholipid Syndrome (APS)(39.58\%), Sjögren Syndrome (SS)(31.25\%), and Rheumatoid Arthritis (RA) (16.67\%). Moreover, in two cases a third AID was registered: SLE-SSAPS and SLE-APS-autoimmune-thyroiditis.

In the SLE-APS group, SLE was the AID of debut in the $89.47 \%$ of cases, instead of SLE-RA group with a $62.5 \%$. The SLE-APS group showed a $47.37 \%$ of cases with positive antiphospholipid antibodies and $64.71 \%$ positive lupus anticoagulant. In the SLE-RA group a $71.43 \%$ and $66.67 \%$ positive rheumatoid factor and antiCCP antibody was reported.

Conclusions $18.39 \%$ of patients with PAI in our group of SLE patients was observed, mostly with the SLE as the first AID developed. The most frequent association of AIDs in SLE cases were with APS, SS and RA.

\section{P63 ABNORMAL DISTRIBUTION OF CD27 ${ }^{+}$IGD $^{+}$ UNSWITCHED AND CD $27^{+}$IGD ${ }^{-}$SWITCHED MEMORY B CELLS IN SLE PATIENTS EXPOSED TO ORGANIC SOLVENTS}

${ }^{1}$ Carolina Hurtado, ${ }^{2}$ Diego Rojas-Gualdrón, ${ }^{2} E l s a$ María Vásquez-Trespalacios, ${ }^{3}$ Ricardo Pineda, ${ }^{4}$ Scott Jenks, ${ }^{5}$ Gloria Vásquez, ${ }^{4}$ Iñaki Sanz. ${ }^{1}$ School of Graduate Studies and School of Medicine, CES University, Medellin, Colombia; ${ }^{2}$ School of Medicine, CES University, Medellin, Colombia; ${ }^{3}$ Group of Clinical Information, Artmedica IPS, Medellin, Colombia; ${ }^{4}$ Dept. of Medicine, Division of Rheumatology, Lowance Center for Human Immunology, Emory University, Atlanta, USA; ${ }^{5}$ Grupo de inmunología celular e inmunogenética, Universidad de Antioquia, Medellin, Colombia

\subsection{6/lupus-2020-eurolupus.110}

Background Some studies in animal models, support an association between occupational exposure to Organic Solvents (OS) and Systemic Lupus Erythematosus (SLE). The specific physiopathological changes that these chemicals could induce to accelerate an autoimmune response are not known. Dysregulation of B cells is central in SLE, but very little is known on how OS exposure could influence it. This study aimed to examine the distribution of $\mathrm{B}$ cell subsets on Healthy Controls and SLE patients occupationally exposed to OS.

Methods 40 SLE patients who met ACR criteria and 17 Healthy Controls were recruited and classified as occupationally exposed or not to OS. Cryopreserved peripheral lymphocytes were analyzed by multiparametric Flow Cytometry using CD3, CD19, CD27, and IgD markers.

Results SLE patients exposed to OS had increased frequencies of CD27+ Switched Memory (SWM) cells. This change was associated with a specific OS like degreasers and ketones. Additionally, the few HC exposed to OS showed a decrease in Unswitched (USM) cells, with similar frequencies as those seen in SLE patients.

Conclusions Exposure to OS increased SWM cells on SLE patients and decreased USM cells on Healthy Controls. The influence of OS on SWM differentiation may be mediated through T cells. Previous reports of exposure to Trichloroethylene (a common OS), showed increased CD4+ T cell activation and secretion of INF- $\gamma$, this causes excessive $\mathrm{T}$ follicular helper development and germinal center formation in mice that could induce abnormalities in B cell subsets, and a similar mechanism may operate in OS exposed patients. Further research is needed to verify this hypothesis.

\section{P66 UTILIZATION OF GEOGRAPHIC INFORMATION SYSTEM (GIS) MAPPING TO ASSESS DISSEMINATION OF A LUPUS COMMUNITY BASED HEALTH AWARENESS MODEL}

${ }^{1}$ Karen Mancera-Cuevas, ${ }^{2}$ Daniel L Erickson, ${ }^{1}$ Anh Chung, ${ }^{2}$ Joan S Chmiel, ${ }^{3}$ Courtnie Phillip, ${ }^{3}$ Candace Feldman, ${ }^{4}$ Patricia Canessa, ${ }^{1}$ Rosalind Ramsey-Goldman. ${ }^{1}$ Medicine/ Rheumatology, Northwestern University, Chicago; ${ }^{2}$ Preventive Medicine, Northwestern University, Chicago; ${ }^{3}$ Division of Rheumatology, Brigham and Womans Hospital, Boston; ${ }^{4}$ Illinois Public Health Association, Springfield, USA

\subsection{6/lupus-2020-eurolupus.111}

Background We used a Popular Opinion Leader (POL) model, which leverages community leaders' social networks to disseminate health information and change norms in vulnerable communities. We established an academic-community partnerships in Chicago and Boston to increase knowledge about lupus and promote early care-seeking behaviors among African American 
individuals. We aimed to describe sociodemographic characteristics of Chicago where POLs disseminated lupus-related information and to explore using the Area Deprivation Index (ADI) as a measure of the sociodemographic characteristics (higher ADI, more deprived).

Methods Fourteen POLs were recruited from primarily African American, underserved areas to disseminate lupus-related information. POLs recorded encounters throughout their social networks noting the number of individuals contacted and the addresses of venues where educational material was shared. GIS mapping documented the networks, the Healthy Chicago Data (HCD) described the sociodemographic factors and health resources of the POL networks, and GIS addresses linked geocodes with the ADI for these neighborhoods by census block tracks.

Results Nine POLs' social networks were concentrated in four selected predominantly African American communities (Washington Heights, Morgan Park, Englewood and Roseland) located on the Southside of Chicago where health, educational, and financial resources are limited using HCD. $8-17 \%$ of individuals living in these areas lacked health insurance, and the percent living below the Federal poverty line and for not completing high school was highest in Englewood $(30.8 \%$, $19.7 \%)$ and Roseland (27.2\%, 13.5\%), respectively. Five additional POLs also participated with social networks in other neighborhoods where we could calculate an ADI as shown in the figure 1. The percentage of ADI encounters in Chicago ranged from 9\% in ADI 1 to 18\% in ADI 10.

Conclusions POLs reached communities with varied ADI categories reflecting dissemination from least to most disadvantaged areas.

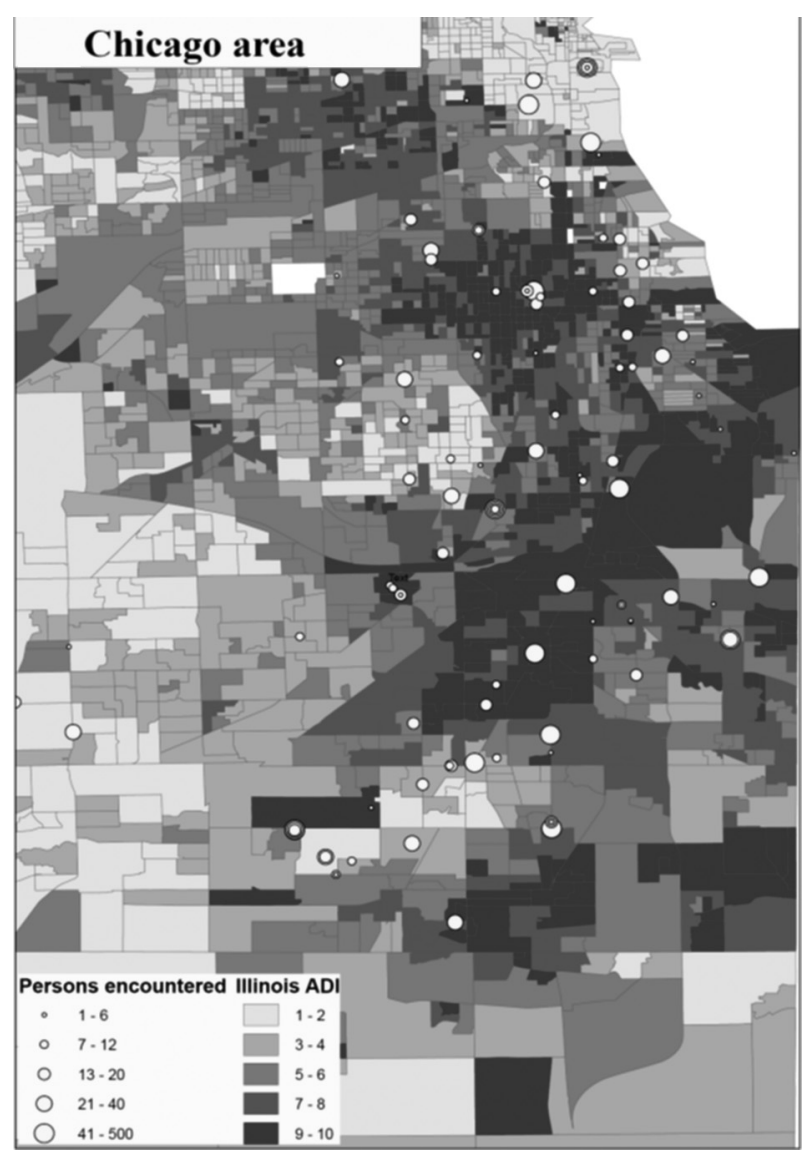

Abstract P66 Figure 1
Acknowledgements Funded by DHHS, OMH Grant \#1CPIMP171141-01-00.

\section{P67 JACCOUD'S ARTHROPATHY IN SYSTEMIC LUPUS ERYTHEMATOSUS: A CASE SERIES OF 52 BRAZILIAN PATIENTS}

Mittermayer Santiago. Rheumatology Service, HUPES/Federal University of Bahia, Salvador, Brazil

\subsection{6/lupus-2020-eurolupus. 112}

Background Jaccoud's arthropathy (JA) is a condition characterized clinically by 'reversible' joint deformities such as swan neck, thumb subluxation, ulnar deviation, 'boutonniere' and hallux valgus, along with an absence of articular erosions on a plain radiograph. JA was initially described in patients with rheumatic fever (RF), but presently the main clinical entity associated to JA is systemic lupus erythematosus (SLE). In general its prevalence in either SLE or RF is around 5\%.

The aim of the present study is to describe a series of SLE patients with JA, followed up at two referral lupus centers in Brazil. Methods The patients included in this study met the SLE classification criteria proposed by the American College of Rheumatology and also presented JA based on previously proposed criteria. Data collection was obtained through interviews, general physical examination with emphasis on the musculoskeletal system and review of medical records. The patients were originated from two SLE referral outpatient clinics in Salvador, Brazil and the search was conducted throughout 2018-2019.

Results Fifty-two patients with JA were included being 51 female, mean age was $48 \pm 13$ years and mean disease duration was $19 \pm 7$ years. The most frequently found joint deformities were swan neck [49/52 (94\%)]; ulnar deviation [32/52 (61\%)] and thumb subluxation [23/52 (44\%)].

Discussion To the best of our knowledge it is the largest case series of JA in SLE ever described. Some patients have been included in previous studies developed by our group. Based on the findings of such studies some conclusions can be drawn: 1) Although in the majority of the cases JA is a 'correctable' arthropathy sometimes the joints are fixed, resembling those seen in rheumatoid arthritis, even in the absence of bone erosions. 2) The level of interleukin 6 is higher in SLE patients with JA as compared to those without such complication. 3) Classically in JA no erosion is seen on plain radiographs, however its presence on magnetic resonance imaging or high-performance ultrasound examination is not so rare.

\section{P68 A DIET RICH IN WHEAT ALPHA-AMYLASE/TRYPSIN INHIBITORS (ATIS) ENHANCES DISEASE PROGRESSION IN THE MRL-FAS(LPR) MOUSE MODEL OF SYSTEMIC LUPUS ERYTHEMATOSUS}

\begin{abstract}
${ }^{1}$ Myriam Meineck, ${ }^{1}$ Simone Boedecker, ${ }^{2}$ Victor Zevallos, ${ }^{1}$ Andreas Schwarting, 1,3 Detlef Schuppan, ${ }^{2} J u$ lia Weinmann-Menke. 'Institute for Translational Immunology and Research Center for Immunotherapy (FZI), Univ. Medical Center, Johannes Gutenberg University Mainz, Mainz, Germany; ${ }^{2}$ Dept. of Nephrology, Johannes-Gutenberg University Mainz, Mainz, Germany; ${ }^{3}$ Division of Gastroenterology, Beth Israel Deaconess Medical Center, Harvard Medical School, Boston, USA
\end{abstract}

10.1136/lupus-2020-eurolupus.113

Background Wheat alpha-amylase/trypsin inhibitors (ATIs) are the second most prevalent proteins in wheat $(3-4 \%$ vs $80-$ 